Preprint typeset in JHEP style. - HYPER VERSION

Cavendish HEP-2001-07

DAMTP-2001-48

ATLAS-COM-PHYS-2001-010

CERN-TH-2001-155

\title{
Extracting the Flavour Structure of a Baryon-Number R-parity Violating Coupling at the LHC
}

\author{
B.C. Allanach*, A.J. Barr ${ }^{\dagger}$, M.A. Parker ${ }^{\dagger}$, P. Richardson ${ }^{\dagger, \dagger}$ and B.R. Webber ${ }^{\dagger}$ \\ * Theory Division, CERN, 1211 Geneva 23, Switzerland. \\ ${ }^{\dagger}$ Cavendish Laboratory, University of Cambridge, Madingley Road, Cambridge, \\ CBз OHE, UK. \\ ${ }^{\ddagger}$ DAMTP, Centre for Mathematical Sciences, Wilberforce Road, Cambridge, \\ CBз $0 W A, U K$.
}

\begin{abstract}
The detection of the flavour content of jets produced from R-parity violating neutralino decays is investigated in the case where one baryon-number violating coupling dominates. Simulations are performed of the ATLAS experiment at the LHC for all couplings, other than $\lambda_{t j k}^{\prime \prime}$ since neutralino decays through these couplings are very suppressed. Secondary vertex distributions and muons produced by heavy-quark (b- and c-) jets allow discrimination between LSP decay modes. The dominant coupling can be identified at better than $3.5 \sigma$ in almost all cases, with the only remaining ambiguity caused by the inability to distinguish strange from down quarks.
\end{abstract}

Keywords: Supersymmetric Standard Model, Hadronic Colliders, Beyond Standard Model, Supersymmetry Breaking. 


\section{Contents}

1. Introduction 1

2. Event Simulation and Selection 3

3. Vertex Tagging 4

4. Flavour Discrimination from Muons 5

5. Statistical Significance 5

6. Conclusions 7

\section{Introduction}

In supersymmetry (SUSY) models where R-parity, $\mathrm{R}_{\mathrm{p}}=(-1)^{3 \mathrm{~B}+\mathrm{L}+2 \mathrm{~S}}$, is violated (RPV models) the lightest supersymmetric particle (LSP) can decay to Standard Model particles. For the baryon-number violating RPV coupling, $\lambda^{\prime \prime}$, the LSP, assumed to be the $\tilde{\chi}_{1}^{0}$, decays via a virtual squark into three quarks (or antiquarks). In previous work [1] we suggested a method by which the masses of several of the SUSY particles can be measured in such scenarios for a wide range of $\lambda^{\prime \prime}$.

If R-parity violating couplings are observed, it seems probable that they come from an underlying theory which also determines the fermion mass spectrum. This idea has been explored [2-5] in the context of gauged $U(1)_{X}$ models of flavour, in which a hierarchical pattern of RPV couplings is generated by the same symmetry from which the fermion mass spectrum is derived. Thus the determination of the flavour structure of an RPV coupling could be a vital clue in the resolution of the flavour problem.

In this paper we investigate the flavour content of the quark jets from $\tilde{\chi}_{1}^{0}$ decays. The aim is to identify which $\lambda_{i j k}^{\prime \prime}$ coupling is dominant in the neutralino decay. The index $i$ is the generation number of an up-type quark, and $j$ and $k$ correspond to down-type quarks. The coupling is antisymmetric in $j$ and $k$, so there are nine possible non-zero elements.

Three of the couplings, $\lambda_{\mathrm{t} j k}^{\prime \prime}$, produce very different signatures because they allow the $\tilde{\chi}_{1}^{0}$ to decay via the top quark. If the neutralino is lighter (heavier) than the top quark then the decay is propagator (phase-space) suppressed. For the mSUGRA 


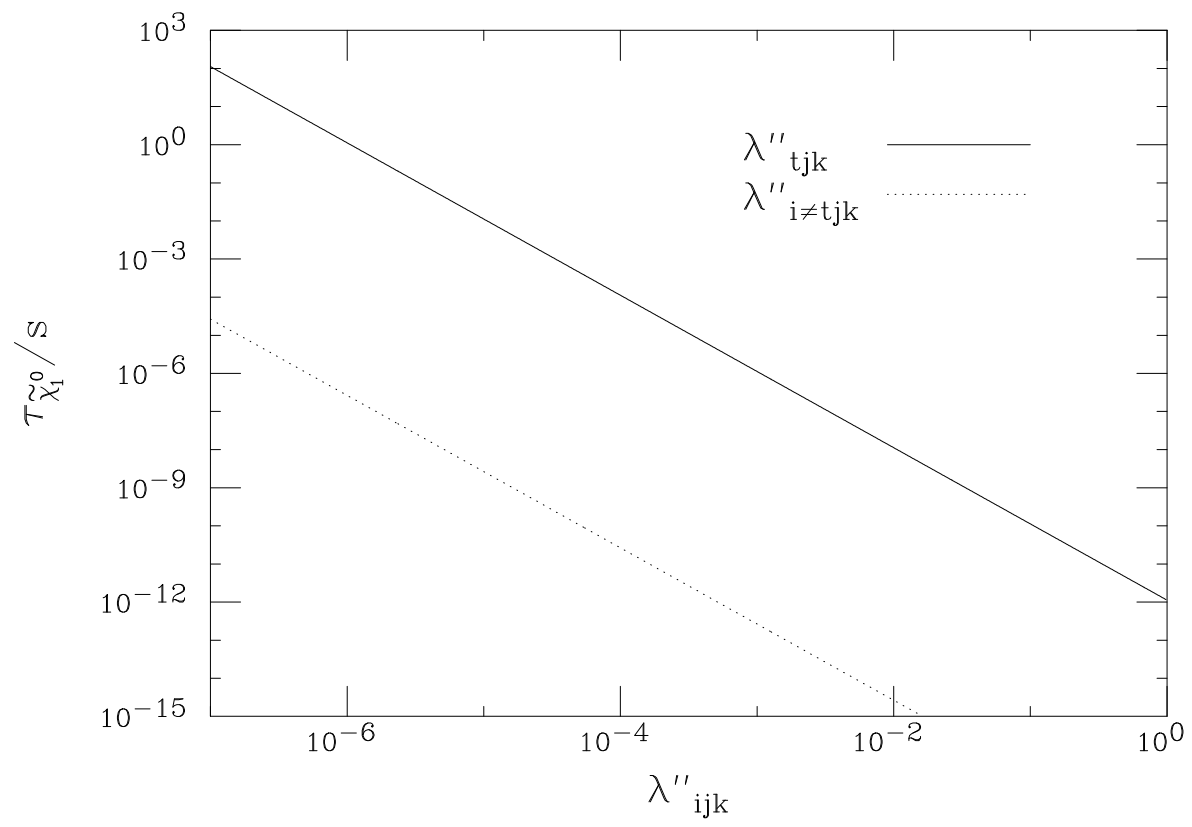

Figure 1: The lifetime of the $\tilde{\chi}_{1}^{0}$ at the mSUGRA point described in the text, with one of $\lambda_{i j k}^{\prime \prime}$ non-zero. The solid line shows the lifetime for a dominant top quark coupling, which leads to the decay $\tilde{\chi}_{1}^{0} \rightarrow \mathrm{qq}^{\prime} \mathrm{t}^{*} \rightarrow \mathrm{qq}^{\prime} \mathrm{bW}^{*} \rightarrow \mathrm{qq}^{\prime} \mathrm{bff}^{\prime}$, where $\mathrm{q}$ and $\mathrm{q}^{\prime}$ are quarks other than top and $f$ and $f^{\prime}$ are Standard Model fermions. The dashed line shows the lifetime for couplings to quarks other than the top. The calculation approximates the final state particles as massless, so that the lines for $\lambda_{\mathrm{t} j k}^{\prime \prime}$ are the same for all $j, k$, as are those for $\lambda_{i j k}^{\prime \prime}$ for all $i \neq \mathrm{t}, j, k$.

point we investigate (which is described in Section 24) the branching ratio through the top coupling is a factor of $4.2 \times 10^{6}$ smaller than through a non-top coupling of the same size.

For a top mode to dominate the decay, a $\lambda^{\prime \prime}$ coupling to top would need to be a factor of at least 2000 greater than any of the non-top couplings. In that case the lightest neutralino would typically be long-lived (Figure 1) and for $\lambda_{\mathrm{t} j k}^{\prime \prime} \lesssim 10^{-2}$ nearly all $\tilde{\chi}_{1}^{0}$ s decay outside the detector. Determination of these couplings could then only be achieved by searching for the rare decay of $\tilde{\chi}_{1}^{0} \mathrm{~s}$ in the active volume, while sparticle mass measurements could be made in the same way as for the $R_{p}$ conserving case [6].

We investigate the other six couplings $-\lambda_{\mathrm{uds}}^{\prime \prime}, \lambda_{\mathrm{udb}}^{\prime \prime}, \lambda_{\mathrm{usb}}^{\prime \prime}, \lambda_{\mathrm{cds}}^{\prime \prime}, \lambda_{\mathrm{cdb}}^{\prime \prime}$ and $\lambda_{\mathrm{csb}}^{\prime \prime}-$ assuming that one is significantly larger than the others. Since the branching ratio is proportional to the square of the coupling, it is sufficient for this analysis that all the subdominant couplings are 4.5 times smaller than the dominant coupling. Then just $5 \%$ of all $\tilde{\chi}_{1}^{0} \mathrm{~s}$ will decay though the subdominant channel, and $90 \%$ of events, each with two $\tilde{\chi}_{1}^{0} \mathrm{~s}$, will contain only dominant-coupling decays. 


\section{Event Simulation and Selection}

HERWIG 6.2 [7-10] is used as the event generator, and the official ATLAS simulation program ATLFAST 2.50 [11], is used to simulate the performance of the ATLAS detector. We use as a test case the mSUGRA point $m_{0}=100 \mathrm{GeV}, m_{1 / 2}=300 \mathrm{GeV}$, $A_{0}=300 \mathrm{GeV}, \tan \beta=10$ and $\operatorname{sgn} \mu+$, with one of $\lambda_{i j k}^{\prime \prime}=5 \times 10^{-3}$. This is in the middle of the range of couplings $10^{-5} \lesssim \lambda^{\prime \prime} \lesssim 1$, for which we can expect double $\tilde{\chi}_{1}^{0}$ decay within the detector, and is approaching indirect limits set from nuclear decays for $\lambda_{\text {uds }}^{\prime \prime}$ and $\lambda_{\text {udb }}^{\prime \prime}$ [12-14]. A selection of the sparticle masses for this point is shown in Table 1. The applicability of our analysis to SUGRA models with other parameters is discussed in our previous work [1]. The method of the event selection, which is also detailed in that paper, is summarised below.

Signal events will contain three jets from each of the two $\tilde{\chi}_{1}^{0}$ s. Since the SUSY cross section is dominated by squark and gluino production, there are usually at least two other jets from the $\mathrm{R}_{\mathrm{p}}$ conserving decays of squarks and gluinos. Requiring the presence of leptons, which can originate in the decay chain $\tilde{\chi}_{2}^{0} \rightarrow \tilde{l}_{R}^{\mp} \ell^{ \pm} \rightarrow \tilde{\chi}_{1}^{0} \ell^{\mp} \ell^{ \pm}$, helps to decrease the Standard Model (SM) background. Events are selected with between eight and ten jets, and with two opposite-sign, same family isolated leptons (e or $\mu$ ).

Cuts on event-shape variables are applied, reducing the SM background to less than $10 \%$ of the SUSY signal, and kinematic cuts are applied to preferentially select jets from neutralino decays. All possible three-jet combinations are inspected and their invariant masses calculated. Since we expect the event to contain two LSP decays we select two of these three-jet combinations with reconstructed masses within $20 \mathrm{GeV}$ of each other as $\tilde{\chi}_{1}^{0}$ candidates. To reconstruct the $\tilde{\chi}_{2}^{0}$ we pick the $\tilde{\chi}_{1}^{0}$ candidate closest in $\eta-\phi$ to either of the leptons from the lepton pair, and find the total invariant mass of the three jets plus those two leptons.

A 2-dimensional Gaussian is fitted to the $\tilde{\chi}_{1}^{0}$ and $\tilde{\chi}_{2}^{0}$ masses, and combinations are selected within $2 \times \sigma$ of that peak. This selection removes much of the combinatoric background, so that the jet multiplicity cut can be relaxed to $8 \leq N_{\text {jet }} \leq 12$. We now have a rather clean sample of events in which we can identify six jets with the two neutralino decays. All $N_{\text {comb }}$ jet combinations which pass the above cuts for any event are accepted and are given weight $1 / N_{\text {comb }}$.

\begin{tabular}{|l|l|l|l|l|l|l|l|l|}
\hline$\tilde{\chi}_{1}^{0}$ & $\tilde{\chi}_{2}^{0}$ & $\tilde{\mathrm{g}}$ & $\tilde{\mathrm{u}}_{R}$ & $\tilde{\mathrm{u}}_{L}$ & $\tilde{\mathrm{d}}_{R}$ & $\tilde{\mathrm{d}}_{L}$ & $\tilde{\ell}_{R}$ & $\tilde{\ell}_{L}$ \\
\hline 116.7 & 211.9 & 706.3 & 611.7 & 632.6 & 610.6 & 637.5 & 155.3 & 230.5 \\
\hline
\end{tabular}

Table 1: Masses of selected particles (in $\mathrm{GeV}$ ) for the model investigated. These were calculated from the mSUGRA parameters using ISASUGRA from the package ISAJET 7.51 [15]. 

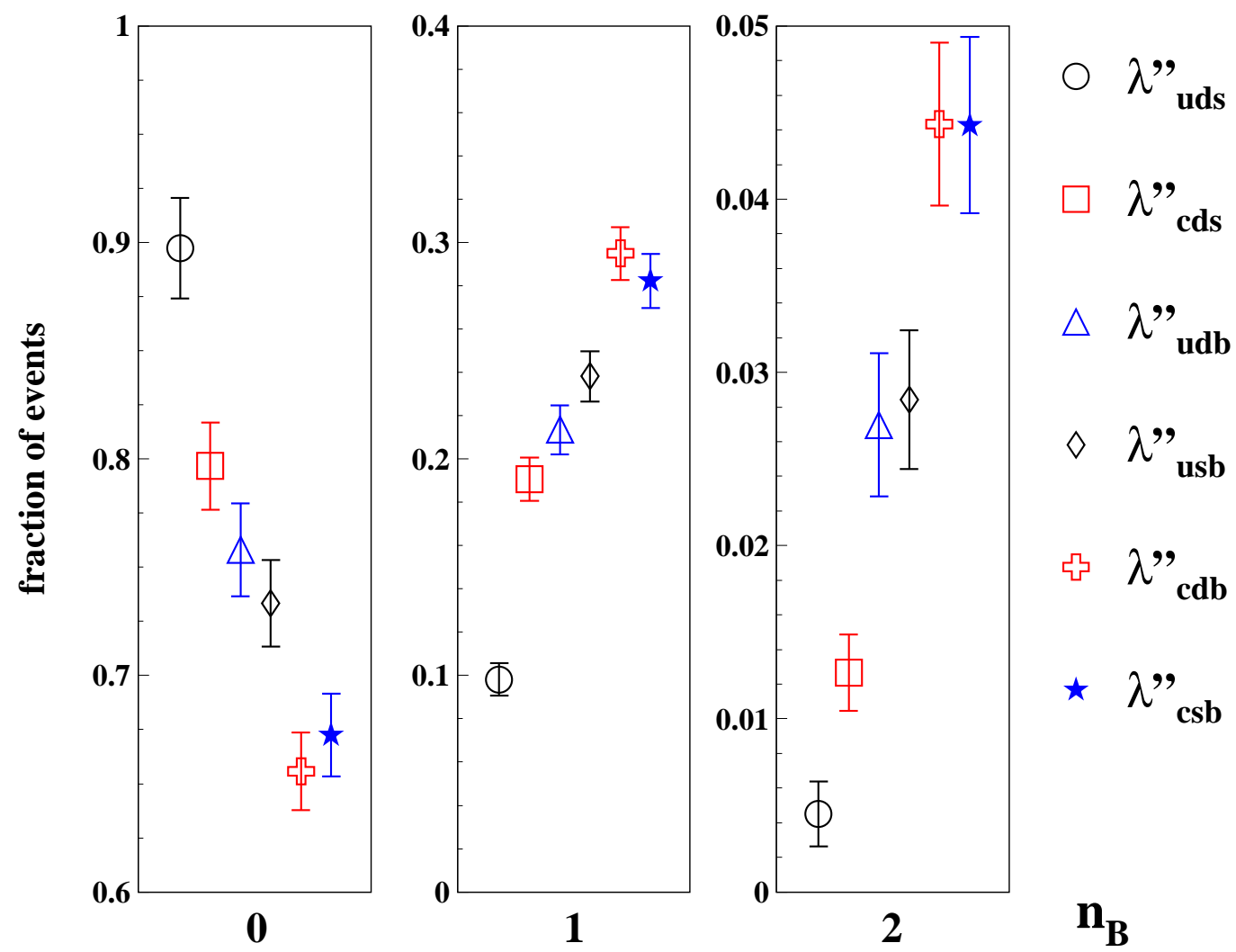

Figure 2: The fraction of signal events with 0,1 , and 2 vertex tags, for different types of RPV coupling $\lambda^{\prime \prime}$. The statistical errors are those expected for an integrated luminosity of $30 \mathrm{fb}^{-1}$. Points are horizontally displaced to allow them to be distinguished by eye.

\section{Vertex Tagging}

The lifetimes of hadrons containing b- and c-quarks give rise to displaced vertices which can be reconstructed from charged tracks in the inner detector. In general the lifetimes of charmed hadrons are shorter than for hadrons containing a b-quark, for example $c \tau_{D^{0}}=124 \mu \mathrm{m}$ compared to $c \tau_{B^{0}}=468 \mu \mathrm{m}$. This allows statistical separation of c-quark jets from b-quark jets, and of $\mathrm{c}$ - and b-jets from light quark $(\mathrm{u}, \mathrm{d}, \mathrm{s})$ and gluon jets. Strange hadrons have longer lifetimes with $c \tau$ of the order of tens of centimeters. Since they decay to a small number of particles it is difficult to reconstruct a secondary vertex, and so strange hadrons cannot be tagged within jets in the LHC environment.

The vertex tagging performance of the ATLAS Inner Detector was simulated in [6]. In that study a likelihood ratio method was applied to the transverse impact parameter $\left(d_{0}\right)$ of the tracks within the jet cone. The resultant b-tagging efficiency and the rejection rates for c- and other jets were parameterised as functions of the transverse momentum and pseudorapidity of the reconstructed jet and were imple- 


\begin{tabular}{|c|c|c|}
\hline b-tagging efficiency & \multicolumn{2}{|c|}{ Rejection factor } \\
\cline { 2 - 3 }$\epsilon_{b}$ & $\mathrm{u}, \mathrm{d}, \mathrm{s}$ and g jets $\left(r_{j}\right)$ & c-jets $\left(r_{c}\right)$ \\
\hline 0.33 & 1400 & 22.9 \\
\hline
\end{tabular}

Table 2: b-tagging efficiencies and mis-tagging rejection factors from a full simulation of the ATLAS inner detector [6]. $1 / r_{c}$ is the probability of tagging a c-jet, while $1 / r_{j}$ is the probability of tagging a $\mathrm{u}, \mathrm{d}, \mathrm{s}$ or gluon jet, averaged over $p_{T}, \eta$ and $\phi$. The typical reconstructed jet $p_{T}$ scale is $50 \mathrm{GeV}$.

mented in ATLFAST. The rejection factors, averaged over all directions and transverse momenta, are summarised in Table 2. In order to select a higher purity sample, we tolerate a rather low b-tagging efficiency (33\%).

The proportions of events with 0,1 and 2 tagged jets are shown in Figure 2, for the six different $\lambda^{\prime \prime}$ couplings. As we expect, the vertex tagging rate is greatest when the $\tilde{\chi}_{1}^{0}$ decay products include both c and b quarks, and smallest for couplings which produce light-quark daughters. As we cannot distinguish d- from s-jets, there is no discrimination between $\lambda_{i \mathrm{db}}^{\prime \prime}$ and $\lambda_{i \mathrm{sb}}^{\prime \prime}($ for $i=\mathrm{u}, \mathrm{c})$.

In these simulations, with $\lambda^{\prime \prime}=5 \times 10^{-3}$ the $\tilde{\chi}_{1}^{0}$ lifetime of $10^{-14}$ seconds corresponds to a typical decay length $c \tau$ of $3 \mu \mathrm{m}$. This is short in comparison with typical b- and c-jet vertex displacements so it should not severely affect vertex reconstruction. However, the lifetime is inversely proportional to the square of the coupling strength. When $c \tau$ becomes bigger than about $3 \mathrm{~cm}\left(\right.$ for $\lambda^{\prime \prime} \lesssim 5 \times 10^{-5}$ ) special vertex reconstruction would be required. Vertex tagging becomes almost impossible when neutralinos travel more than about $30 \mathrm{~cm}$ in the transverse direction since their daughters will produce hits only in the outermost silicon layers.

\section{Flavour Discrimination from Muons}

The weak decay of hadrons containing heavy quarks can produce muons, which will generally lie within the associated quark jet. The muons will pass though the calorimeter and so can be measured by the muon detector even if they lie inside the jet cone. The frequency with which these "non-isolated" muons occur in signal events can be used as an additional discriminator between quark flavours.

The number of non-isolated muons per event in the six signal jets is plotted in Figure 3. Since bottom mesons produce more muons $\left[B\left(B^{0} \rightarrow \mu^{+} \nu_{\mu} \mathrm{X}\right)=10 \%\right]$ than charmed mesons $\left[B\left(D^{0} \rightarrow \mu^{+} \nu_{\mu} \mathrm{X}\right)=7 \%\right][16]$, we can use the number of muons in signal jets to statistically separate b- from c-jets.

\section{Statistical Significance}

The confidence with which we can identify the dominant coupling was explored for all pairs of couplings for both the vertex tagging and muon rates (Figures 2 and 3 

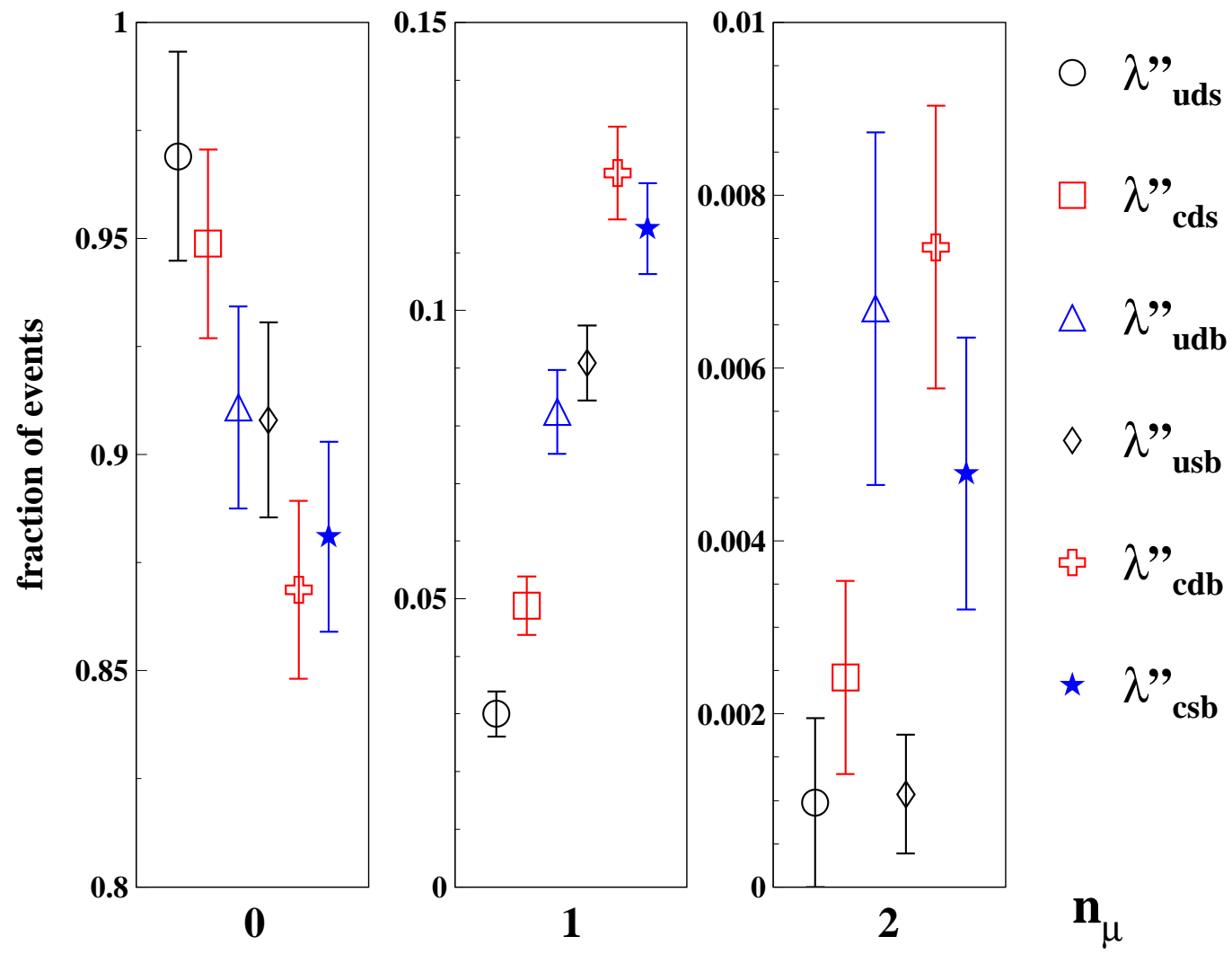

Figure 3: The fraction of signal events with 0,1 and 2 muons that lie within $\sqrt{\Delta \eta^{2}-\Delta \phi^{2}} \leq 0.4$ of the signal jets, for different types of RPV coupling, $\lambda^{\prime \prime}$. The statistical errors are those expected for an integrated luminosity of $30 \mathrm{fb}^{-1}$. Points are horizontally displaced to allow them to be distinguished by eye.

respectively). The variable,

$$
\chi^{2}=\sum_{i} \frac{\left(x_{i}-y_{i}\right)^{2}}{\sigma_{x}^{2}+\sigma_{y}^{2}}
$$

was calculated, where $x_{i}$ and $y_{i}$ are the fractions of events with $i$ muons (or vertex tags) for the test pair of couplings and the $\sigma^{2}$ s are their variances assuming Poissondistributed numbers of events. Bins where one or other distribution contained fewer than five events were excluded.

The calculated $\chi^{2}$ values for both the muon and vertex-tagging plots are shown in Table 3. If we use only the muon information then all couplings are distinguishable at $90 \%$ except for the ambiguity between d- and s-jets. In general the vertexing rate gives better discrimination, but would be difficult for a long-lived $\tilde{\chi}_{1}^{0}$ for the reasons discussed in Section 3. Combining the results from both analyses gives separation at better than $3.5 \sigma$ in all cases, again with the exception that it is not possible to distinguish down from strange quarks. 


\begin{tabular}{|c|c|c|c|c|c|c|}
\hline \multicolumn{2}{|c|}{\begin{tabular}{l} 
Distinguishing \\
\multicolumn{2}{|c|}{$\lambda_{i j k}^{\prime \prime}$ from $\lambda_{l m n}^{\prime \prime}$}
\end{tabular}} & \multicolumn{2}{|c|}{ Vertexing } & \multicolumn{2}{c|}{ Muons } & Combined \\
\cline { 2 - 5 } uds & $\chi^{2} /$ d.f. & $\mathrm{P} / \%$ & $\chi^{2} /$ d.f. & $\mathrm{P} / \%$ & discrimination $/ \sigma$ \\
& $\mathrm{udb}$ & $59.1 / 1$ & - & $28.7 / 1$ & - & 9.4 \\
& $\mathrm{usb}$ & $73.0 / 1$ & - & $31.7 / 1$ & - & 10.2 \\
& $\mathrm{cds}$ & $30.5 / 1$ & - & $4.0 / 1$ & 4 & 5.9 \\
& $\mathrm{cdb}$ & $106.9 / 1$ & - & $47.2 / 1$ & - & 12.4 \\
& $\mathrm{csb}$ & $113.4 / 1$ & - & $49.2 / 1$ & - & 12.8 \\
\hline $\mathrm{udb}$ & $\mathrm{usb}$ & $1.6 / 2$ & 44 & $0.4 / 1$ & 54 & 1.4 \\
& $\mathrm{cds}$ & $10.3 / 2$ & 1 & $13.0 / 1$ & - & 4.8 \\
& $\mathrm{cdb}$ & $18.3 / 2$ & - & $6.8 / 2$ & 3 & 5.0 \\
& $\mathrm{csb}$ & $16.3 / 2$ & - & $5.1 / 2$ & 8 & 4.6 \\
\hline $\mathrm{usb}$ & $\mathrm{cds}$ & $17.5 / 2$ & - & $17.2 / 1$ & - & 5.9 \\
& $\mathrm{cdb}$ & $12.1 / 2$ & - & $5.1 / 1$ & 2 & 4.2 \\
& $\mathrm{csb}$ & $9.9 / 2$ & 1 & $3.1 / 1$ & 8 & 3.6 \\
\hline $\mathrm{cds}$ & $\mathrm{cdb}$ & $56.1 / 2$ & - & $37.4 / 1$ & - & 9.7 \\
& $\mathrm{csb}$ & $55.8 / 2$ & - & $35.3 / 1$ & - & 9.5 \\
\hline $\mathrm{cdb}$ & $\mathrm{csb}$ & $0.6 / 2$ & 72 & $1.3 / 2$ & 51 & 1.4 \\
\hline
\end{tabular}

Table 3: Chi-squared function, and number of degrees of freedom (d.f.) for the difference in distributions for pairs of RPV couplings $\left(\lambda^{\prime \prime}\right)$ for an integrated luminosity of $30 \mathrm{fb}^{-1}$. The contributions from vertexing and muon counting are shown separately. The probability $(\mathrm{P})$ in the tail of the chi-squared distribution is given when $\mathrm{P} \geq 1 \%$. The number of degrees of freedom is one less than the number of histogram bins in which both couplings contain at least five events.

If the branching ratios of the neutralino through two or more couplings were comparable, then as the information in the distributions is rather degenerate, definitive identification of the couplings becomes difficult. However the method could then be used to constrain the possible values of the couplings.

\section{Conclusions}

If baryon-number violating couplings to quarks other than top are $\gtrsim 5 \times 10^{-5}$ then $\tilde{\chi}_{1}^{0} \mathrm{~s}$ will typically decay within the tracking volume of an LHC detector. For these couplings, our simulations show that displaced secondary vertices and muons from heavy quark decays allow statistical separation of b- from c-quark jets, and b- and c-jets from light-quark jets. A dominant RPV coupling can then be identified at better than $3.5 \sigma$, except for an ambiguity caused by the inability to distinguish strange from down quarks.

We have demonstrated that if the MSSM breaks baryon number, not only can the LHC detect and measure the masses of sparticles [1], but it can even distinguish the flavour structure of the RPV coupling. 


\section{Acknowledgments}

We would like to thank F. Paige for suggesting this extension of our previous work. We thank H. Dreiner, members of the Cambridge SUSY working group, and the ATLAS collaboration for helpful discussions. We have made use of the physics analysis framework and tools which are the result of collaboration-wide efforts. This work was partly funded by PPARC.

\section{References}

[1] B. C. Allanach et. al., Measuring Supersymmetric Particle Masses at the LHC in Scenarios with Baryon-Number R-parity Violating Couplings, JHEP 03 (2001) 048, hep-ph/0102173.

[2] J. Ellis, S. Lola, and G. G. Ross, Hierarchies of R-violating Interactions from Family Symmetries, Nucl. Phys. B526 (1998) 115, hep-ph/9803308.

[3] J. L. Chkareuli, I. G. Gogoladze, A. B. Kobakhidze, M. G. Green, and D. E. Hutchcroft, On SUSY Inspired Minimal Lepton Number Violation, Phys. Rev. D62 (2000) 015014, [hep-ph/9908451].

[4] G. K. Leontaris and J. Rizos, New Fermion Mass Textures from Anomalous U(1) Symmetries with Baryon and Lepton Number Conservation, Nucl. Phys. B567 (2000) 32, hep-ph/9909206.

[5] J. Ellis, G. K. Leontaris, and J. Rizos, Implications of Anomalous U(1) Symmetry in Unified Models: The Flipped SU(5)×U(1) Paradigm, JHEP 05 (2000) 001, hep-ph/0002263.

[6] ATLAS Detector and Physics Peformance TDR. CERN, 1999.

[7] G. Marchesini et. al., HERWIG: A Monte Carlo Event Generator for Simulating Hadron Emission Reactions with Interfering Gluons. Version 5.1 - April 1991, Comput. Phys. Commun. 67 (1992) 465.

[8] G. Corcella et. al., HERWIG 6: An Event Generator for Hadron Emission Reactions with Interfering Gluons (including Supersymmetric Processes), JHEP 01 (2001) 010, hep-ph/0011363.

[9] H. Dreiner, P. Richardson, and M. H. Seymour, Parton-shower Simulations of R-parity Violating Supersymmetric Models, JHEP 04 (2000) 008, hep-ph/9912407.

[10] P. Richardson, Simulations of R-parity Violating SUSY Models, hep-ph/0101105.

[11] E. Richter-Was, D. Froidevaux, and L. Poggioli, ATLFAST 2.0: A Fast Simulation Package for ATLAS, ATL-PHYS-98-131. ATLAS internal note. 
[12] M. Sher and J. L. Goity, Bounds on $\Delta B=1$ Couplings in the Supersymmetric Standard Model, hep-ph/9503472.

[13] J. L. Goity and M. Sher, Bounds on $\Delta B=1$ Couplings in the Supersymmetric Standard Model, Phys. Lett. B346 (1995) 69-74, hep-ph/9412208.

[14] B. C. Allanach, A. Dedes, and H. K. Dreiner, Bounds on R-parity Violating Couplings at the Weak Scale and at the GUT Scale, Phys. Rev. D60 (1999) 075014, hep-ph/9906209.

[15] H. Baer, F. E. Paige, S. D. Protopopescu, and X. Tata, ISAJET 7.48: A Monte Carlo Event Generator for $p$, $\bar{p} p$, and $e^{+} e^{-}$Reactions, hep-ph/0001086.

[16] C. Caso et. al., Review of Particle Physics. Particle Data Group, Eur. Phys. J. C3 (1998) 1-794. 